\title{
Gingival Recession Associated with Orthodontic Treatment and Root Coverage
}

\author{
Alparslan Dilsiz ${ }^{1}$, Tugba Aydin ${ }^{2}$ \\ ${ }^{1}$ DDS, PhD. Assistant Professor, Department of Periodontology, Faculty of Dentistry, Atatürk University, Erzurum/ TURKEY. \\ ${ }^{2}$ DDS, Post Graduate Student, Department of Periodontology, Faculty of Dentistry, Atatürk University, Erzurum/ TURKEY.
}

Correspondence:

Alparslan DILSIZ, DDS, PhD.

Assistant Professor,

Department of Periodontology,

Faculty of Dentistry, Atatürk University,

25240-Erzurum/TURKEY

E-mail:aydilsiz@yahoo.com,dtkoseoglu@hotmail.

\begin{abstract}
Many patients seek to pursue orthodontic treatment for esthetic improvement. These patients present with malalignment of the anterior teeth. There was strong correlation between the severity and extent of gingival recessions and past orthodontic treatment, it was suggested that orthodontic tooth movement may lead to gingival recession. The principal objective in the treatment of gingival recession is to cover the exposed root surfaces to improve esthetics and to reduce hypersensitivity. Different soft tissue grafting procedures have been proposed in the treatment of gingival recessions. Free gingival grafts (FGG) are a reliable method for treatment of gingival recessions: An autograft is taken from the palate as replacement for the lost keratinized gingiva. The purpose of this case report was to illustrate the relationship between orthodontic therapy and gingival recession, and to describe the successful treatment of this case. A 24-year-old girl with gingival recession and hypersensitivity in the anterior mandibulary region was admitted to periodontology clinic. Treatment consisted of oral hygiene instruction, mechanical debridement and surgical periodontal treatment. Root coverage is performed with a FGG in a one step method. The patient and clinicians were satisfied with the result. FGG can a viable alternative in the treatment of gingival recession. Surgical periodontal treatments result in esthetic improvement, elimination of sensitivity, and less risk of developing root caries.
\end{abstract}

Key words: Gingival recession, etiology, orthodontic, treatment, free gingival graft. 


\section{Introduction}

Gingival recession is the most common and undesirable condition. It is characterized by the displacement of the gingival margin apically from the cemento-enamel junction (CEJ) and the exposure of the root surface to the oral environment. For a patient, gingival recession usually creates an aesthetical problem and fear of tooth loss due to progressing destruction, and it may also be associated with dentine hypersensitivity and/or root caries, and cervical wear.

The etiology of gingival recession is multifactorial. Several factors may play a role in recession development, i.e., excessive or inadequate teeth brushing; destructive periodontal disease; tooth malpositioning; alveolar bone dehiscence; thin and delicate marginal tissue covering a nonvascularized root surface; high muscle attachment and frenal pull; occlusal trauma; lip piercing; and iatrogenic factors related to reconstructive, conservative periodontologic, orthodontic, or prosthetics treatment (1). Among these etiologic factors, a strong correlation was found between the severity and extent of gingival recession to past orthodontic treatment, and it was suggested that orthodontic tooth movement, especially beyond the labial or lingual alveolar plate, may lead to gingival recession (2).

The main indications for root coverage procedures are esthetic and/or cosmetic demands, followed by the management of root hypersensitivity, shallow root caries lesions, and cervical abrasions. Coverage of denuded roots has become one of the most challenging procedures in periodontal mucogingival surgery (1). The search for the appropriate root coverage technique has taken many different approaches. Various surgical options have been developed to achieve the above goals and include the use of FGGs, subepithelial connective tissue grafts, laterally sliding flaps, coronally advanced flaps, double papilla flaps, guided tissue regeneration, and acellular dermal matrix allografts (3-7).

Since it was described in 1963 (3), the FGGs had been utilized to increase amounts of keratinized tissue and obtain root coverage. This extremely predictable procedure has been used to treat various types of mucogingival defects, including recession, the aberrant frenum, and the shallow vestibule. However, probably the procedure's most common use is to increase the amount of keratinized tissue. When utilized for augmentation, FGG is a procedure with a very high degree of predictability $(8,9)$. The adaptability of the FGG was demonstrated by its widespread use in root coverage procedures (4).

The purpose of this case report was to illustrate the relationship between orthodontic therapy and gingival recession, and to describe the successful treatment of this case with FGG.

\section{Case report}

A 24-year-old girl was referred by her orthodontist to the periodontology clinic of the Faculty of Dentistry, Atatürk University, for evaluation and treatment of the gingival recession associated with the mandibular right incisor.

Her general health condition was good, did not take any medications, had no known allergies and was nonsmoker. The dental history revealed that she had undergone orthodontic therapy in both arches between 19 and 22 years of age. Since that time she received routine general dental care and maintenance twice each year. Her chief complaint was concern relative to the recession associated with the lower front tooth. The patient reported that she first noticed the recession about 8 months ago, that it was getting progressively worse, and was sensitive to tooth-brushing. She was excited about attempting to eliminate the esthetic problem.

Clinical evaluation revealed gingival recession on the buccal surface extending $4 \mathrm{~mm}$ apical to the CEJ and a narrow zone of attached gingival measuring approximately $1 \mathrm{~mm}$ (Fig.1). There was no loss of papilla height on the mesial and distal aspect of the incisor. No plaque accumulation was detected in the affected site. There was no gingival recession associated with adjacent teeth. The gingiva in these regions had health appearance. The patient's oral hygiene was good. There was no bone loss on the mesial and distal aspects of the affected tooth.

The aim of the treatment was to restore harmonious appearance of the gingival by covering the root surface to an equal level of the neighboring teeth and, at the same time, to increase the zone of attached gingiva. It was decided to treat this problem with a FGG.

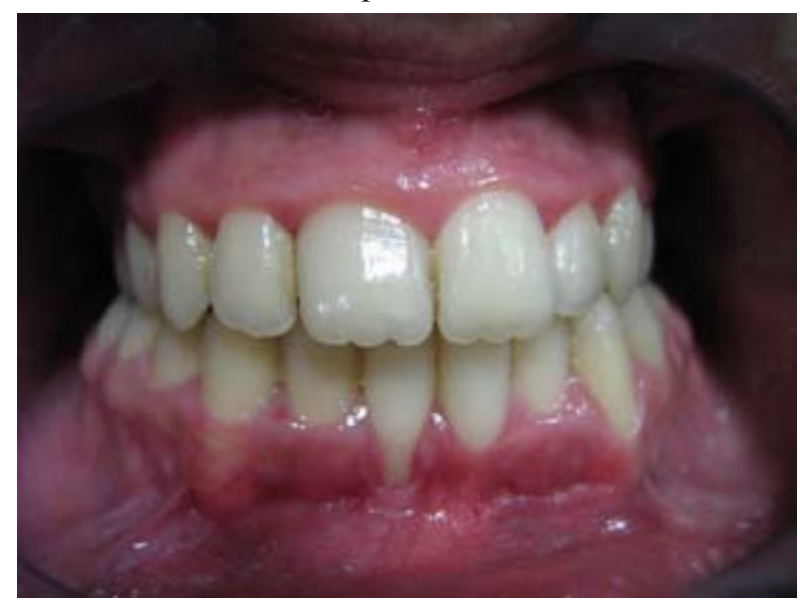

Fig.1. Preoperative view

Written informed consent was obtained from the patient after all treatment procedures had been fully explained. Before surgical treatment of the gingival recession, the patient underwent crown polishing and oral hygiene instruction.

Surgical procedure

The exposed root surface was carefully planed with the 
use of Gracey curettes. The area was gently irrigated with sterilized physiological saline solution. Before surgery, extraoral antisepsis was performed with $10 \%$ povidoneiodine solution. The defect was treated by approach described by Miller (4). A local anesthetic was administered to donor and recipient sites to achieve anesthesia.

Following local anesthesia, for preparing a recipient bed for the FGG, a horizontal incision was made at the level of the CEJ in correspondence to the base of adjacent papilla to the line angles of the neighboring teeth from which vertical incisions were realized to the apical extent of the recipient bed, $3 \mathrm{~mm}$ apical to the recession, such delimited tissue was then removed by a partial thickness incision. The graft of adequate size and of about $1 \mathrm{~mm}$ thickness was then harvested from the palate in correspondence to the bicuspid area by a partial thickness incision with a no.15 blade; within 1 min of removal, the graft was then sutured to the recipient bed by three lateral 4-0 silk sutures and was then stabilized by a tooth-suspended 4-0 silk crossed suture (Fig.2); an attempt was made to place the coronal margin of the graft in correspondence to the CEJ. The grafted tissue was then compressed for about 5 min onto the recipient bed. The donor and recipient sites were protected with periodontal pack.

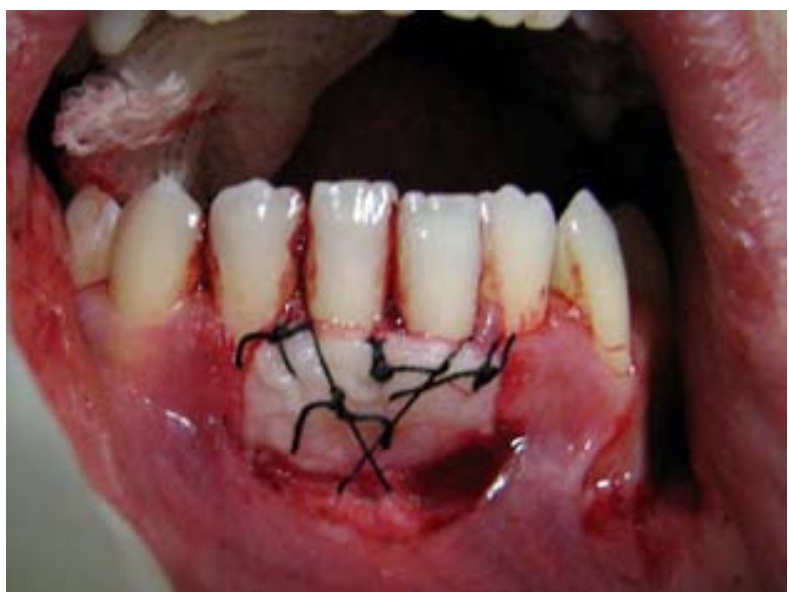

Fig. 2. Free gingival graft sutured in place. The roots are covered to the level of the CEJ.

The patient was prescribed analgesics and instructed to rinse twice daily with $0.12 \%$ chlorhexidine rinse for 2 weeks postoperatively and to avoid trauma or pressure at the surgical site. Toothbrushing activities in the operated sites were discontinued during this time. The dressing and sutures were removed 10 days after surgery, home care instructions were given. Professional prophylaxis was done weekly for the first month and then at 4-month interval.

\section{Clinical Observations}

Four months following surgery, the affected area had completely healed. Probing depth at the midbuccal site was less than $1 \mathrm{~mm}$, the free gingival margin was located less than $1 \mathrm{~mm}$ apically to the CEJ, and the amount of attached gingiva was adequate (Fig.3). The patient's plaque control was good, although moderate tooth staining was apparent.

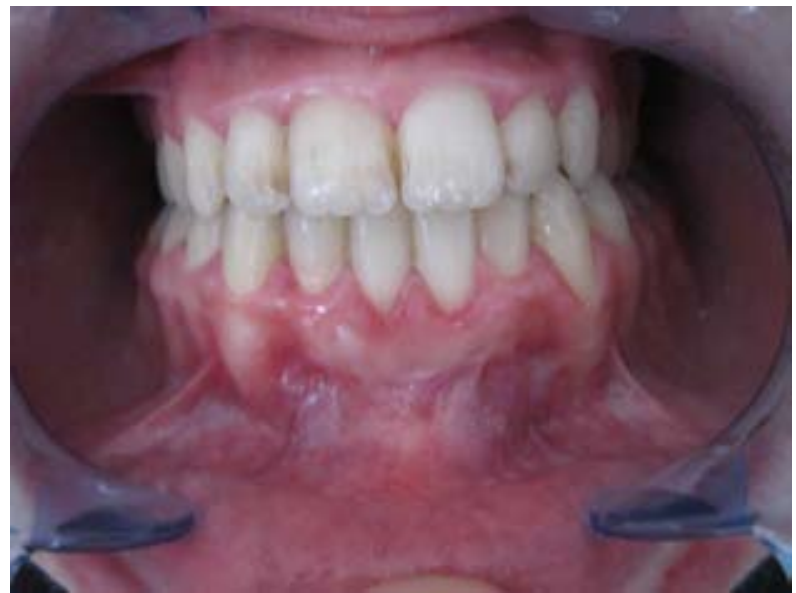

Fig. 3. Clinical view at 4 months post-surgery. Normal appearance was established, with a significant increase of keratinized attached gingiva and a free gingival margin that is in harmony with the neighboring teeth.

The patient was followed for 12 months postoperatively and complete tissue healing was achieved. The patient reported at her postoperative appointment that the final esthetics, both color match and tissue contours, were acceptable to her.

\section{Discussion}

Periodontitis and tooth-brushing trauma are considered the most significant factors causing gingival recession, particularly when associated with predisposing factors such as thin gingival, prominent root surface, buccally positioned teeth, and bone dehiscences (1). The case described here demonstrates that past orthodontic treatment is a potential cause of gingival recession and attachment loss.

Typically, gingival recessions result in an asymptomatic onset and develop slowly over time. Sometimes, patients complain of dentin hypersensitivity and a worsening of esthetic appearance with subsequent psychological discomfort. In the present case, there were tooth hypersensitivity and esthetic problem of the patient.

Soft tissue augmentation procedures aimed at increasing the zone of attached gingiva, as well as covering root surfaces that become exposed because of gingival recession when they cause functional or aesthetic trouble, have been routinely performed using masticatory mucosal autografts. One of the soft tissue augmentation procedures for root coverage is the FGG (4). FGGs have been utilized to increase amounts of keratinized tissue and obtain root coverage, considered necessary to improve the marginal adaptation of soft tissue to the root surfaces and to inhibit further apically-directed loss of soft tissues and bone $(3,4)$. 
with $\%$ s of root coverage ranging from $11 \%$ to $100 \%$ (10-12). These differences may be attributed to differences in the severity of gingival recessions and in surgical techniques, i.e., the use of root conditioning agents and the thickness of the grafted tissue. The successful root coverage with $\mathrm{FGG}$ was obtained \%s ranging from 90 to $100 \%$ in class 1 and 2 gingival recession, as was demonstrated in this case (12). The presence of a wide band of keratinized gingiva was known to provide better plaque control, which leads to a possible significant improvement of the periodontal attachment apparatus (13). The FGG is a favorable alternative to obtain satisfying amounts of keratinized tissue, as was showed in this case.

With the increase in popularity of root coverage procedures, esthetics have become more important in periodontal therapy. Unfortunately, the esthetics of these procedures undertaken to increase keratinized tissue were often less than ideal. Therefore, graft thickness should be considered as an important criteria and should be controlled carefully. While a graft thickness of $0.9 \mathrm{~mm}$ is functionally sufficient on a periosteal bed, thickness of 1.0 to $1.5 \mathrm{~mm}$ was reported as optimal dimensions of the transplanted tissue (14). On the other hand, very thin grafts ( 0.5 to $0.6 \mathrm{~mm}$ thickness $)$ demonstrate a better color blending with that of the neighboring tissues (15). The final esthetics, both color match and tissue contours, were acceptable to the patient in our case.

In conclusion, FGG can a viable alternative in the treatment of gingival recession. The surgical procedure is technique-sensitive and attention to the details involved in the execution of the surgery is crucial to achieve a successful and satisfying outcome. Surgical periodontal treatments result in esthetic improvement, elimination of sensitivity, and less risk of developing root caries.

\section{References}

1. Greenwell H, Fiorellini J, Giannobile W, Offenbacher S, Salkin L, Townsend C, et al; Research, Science and Therapy Committee. Oral reconstructive and corrective considerations in periodontal therapy. $\mathrm{J}$ Periodontol. 2005;76:1588-600.

2. Slutzkey S, Levin L. Gingival recession in young adults: occurrence, severity, and relationship to past orthodontic treatment and oral piercing. Am J Orthod Dentofacial Orthop. 2008;134:652-56.

3. Nabers JM. Free gingival grafts. Periodontics. 1966;4:243-5.

4. Miller PD Jr. Root coverage with the free gingival graft. Factors associated with incomplete coverage. J Periodontol. 1987;58:674-81.

5. Langer B, Langer L. Subepithelial connective tissue graft technique for root coverage. J Periodontol. 1985;56:715-20.

6. Bernimoulin JP, Luscher B, Muhlemann HR. Coronally repositioned periodontal Flap. J Clin Periodontol. 1968;39:65-7.

7. Tal H, Moses O, Zohar R, Meir H, Nemcovsky C. Root coverage of advanced gingival recession: A comparative study between acellular dermal matrix allograft and subepithelial connective tissue grafts. J Periodontol. 2002;73:1405-11.

8. Dorfman HS, Kennedy JE, Bird WC. Longitudinal evaluation of free autogenous gingival grafts. A four year report. J Periodontol. 1982;53:349-52.

9. Edel A. Clinical evaluation of free connective tissue grafts used to increase the width of keratinised gingiva. J Clin Periodontol.
1974;1:185-96.

10. Matter J. Creeping attachment of free gingival grafts. A five year follow-up study. J Periodontol. 1980;51:681-5.

11. Roccuzzo M, Bunino M, Needleman I, Sanz M. Periodontal plastic surgery for treatment of localized gingival recessions: a systematic review. J Clin Periodontol. 2002;29:178-94.

12. Miller PD Jr. Regenerative and reconstructive periodontal plastic surgery. Mucogingival surgery. Dent Clin North Am. 1988;32:287306.

13. Camargo PM, Melnick PR, Kenney EB. The use of free gingival grafts for aesthetic purposes. Periodontol 2000. 2001;27:72-96.

14. Maynard JG Jr. Coronal positioning of a previously placed autogenous gingival graft. J Periodontol. 1977;48:151-5.

15. Lampert F, Lange DE, Flores de Jacoby L. Free mucosal transplantations. Dtsch Zahnarztl. 1976;31:367-70. 